\title{
Sector based Multi-hop Clustering Protocol for Wireless Sensor Networks
}

\author{
Bore Gowda S B \\ Assistant Professor \\ Dept of $E$ and $C$ \\ MIT \\ Manipal University \\ Manipal, India-576104
}

\author{
Puttamadappa C \\ Principal \\ Sapthagiri College of \\ Engg. \\ Karnataka \\ Bangalore \\ India - 560057
}

\author{
Mruthyunjaya H S \\ Professor \\ Dept. of $E$ and $C$ \\ MIT \\ Manipal University \\ Manipal, India-576104
}

\author{
Babu N V \\ Assistant Professor \\ Dept. of $E$ and C \\ SJBIT \\ VTU \\ Bangalore- 560060
}

\begin{abstract}
Minimization of energy consumption is a critical design requirement for most wireless sensor networks (WSNs) applications. WSNs are composed of small, energy constrained sensor nodes. The random deployment of sensor nodes in many applications requires unattended operation. The primary goal of WSNs is how to collect sensed information in energy efficient way since the energy is limited. The network lifetime is extended by hierarchical network clustering. In these networks, the sensor field is partitioned into small regions called clusters. Some of the nodes in the network become cluster head $(\mathrm{CH})$, which aggregates the data collected from their member nodes, and transmit it to the base station using multi-hop communication. Numerous clustering protocols have been proposed in the literature to minimize the energy consumption among the sensor nodes. In this paper, an energy efficient clustering protocol for WSNs with a primary goal of increasing the lifetime of the network is proposed. The protocol named as sector based multi-hop clustering (SBMC), which uses the concept of leveling, sectoring and multi-hop clustering. Dissemination of data from $\mathrm{CH}$ to base station is done in such a way that the energy loss in discovering a route is decreased to minimum. The additional computation for the leveling and sectoring are carried out in base station just at the beginning of the network setup. Therefore, the goal of energy efficiency without additional energy consumption of the distributed nodes after network setup process in proposed SBMC protocol is achieved.
\end{abstract}

\section{Keywords}

Wireless sensor networks, Energy efficient, Leveling, Sectoring, Energy conservation, Multi-hop

\section{INTRODUCTION}

The rapid technological advances in the field of communication, wireless communication have become ubiquitous due to the distributed capabilities, freedom, and cost savings they offer. Wireless sensor networks have emerged as the next wave of wireless technology, enabling greatly distributed measurements across large physical systems. A WSN is a self-configuring network of small sensor nodes communicating among them using radio signals. These nodes may be deployed manually or randomly to sense what is happening in the physical world and report the sensor readings to a processing place i.e. base station where appropriate decisions are taken about the environment being controlled. Currently, WSNs are employed in applications [13] such as habitat monitoring, disaster relief, target tracking, ranging from forest fire detection to soil moisture level testing, military to medical field, from home automation to industry, from safety of large building to bridges and many more.

A WSN consists of large number (order of hundreds or thousands) of low cost sensor nodes which could either have a fixed position or randomly deployed to monitor the environment. The sensed data transmission ends at main collecting center called base station (sometimes they are referred as sinks). The base station collects, filters and aggregates the data sent by sensor nodes in order to extract useful information. In some of the application base station links the sensor network to another network i.e. internet to disseminate the data sensed for further processing. Base stations have enhanced capabilities over simple sensor nodes in terms of memory, energy and computational power to perform complex data processing and their tasks well. Due to size and cost constraints, sensor nodes are equipped with small batteries that can store small power [4]. This puts significant constraints on the power available for communications, thus limiting the transmission range and data rate. A Sensor node in such a network usually communicate directly only with other sensor nodes that are within a small communication range. To enable communication between sensor nodes not within each other's communication range or with the base station, the sensor nodes employ multi-hop data routing approach, which is more energy-efficient than singlehop communication.

Unlike in wired networks or in mobile and cellular networks, energy conservation in WSNs is a critical issue that has been addressed by substantial research works. The energy saving is an open challenging issue in WSNs. In the literature, the energy conservation in sensor nodes is dealt at different levels:

a. Data aggregation to reduce the amount of redundant data transmitted

b. Efficient scheduling of sensor states i.e. sleep and active

c. Efficient transmission power control 
d. Energy aware medium access protocol for channel access and packet transmission

e. Energy efficient clustering and routing data using multi-hop communication

Clustering the WSNs has been proven energy efficient [57]. The sensor nodes in the network are organized into number of small groups called clusters. Each cluster has a coordinator, called cluster head and the number of member nodes. Clustering the WSNs results in a two-tier hierarchy, in which the $\mathrm{CHs}$ form the upper tier manage the grouped lower level nodes i.e. member nodes and the member nodes form the lower tier. The member nodes report their data to the respective $\mathrm{CHs}$. The $\mathrm{CH}$ processes, filters and compresses data sent by member nodes and send them to the central base station through intermediate $\mathrm{CHs}$ [9-12]. Since the $\mathrm{CHs}$ have responsibility for collecting, aggregating and transmitting data over longer distances to the base station, they consume more energy compared to member nodes. The sensor network may be re-organized to form new clusters periodically to select energy-abundant nodes as $\mathrm{CHs}$, thus distributing the energy load uniformly among all the nodes. In addition to energy efficiency, clustering reduces channel contention and packet collisions.

Network lifetime is a primary metric for evaluating the performance of a sensor network. Cluster based routing protocols have been shown to improve network lifetime. The major advantage of clustered WSNs has following advantages over non-clustered networks:

i. Capable of reducing the volume of intra cluster communication by localizing data transmission within the clusters and decreasing the overall number of transmissions to the base station.

ii. Capable of extending the nodes sleep times by allowing $\mathrm{CHs}$ to coordinate and optimize the activities of member nodes through some form of TDMA based scheduling.

iii. Provide scalability and robustness for the network.

The rest of the paper is organized as follows. Section 2 provides an overview of related work on clustering. Section 3 describes the network model and necessary preliminaries of the work. In Section 4 a new energy efficient clustering protocol is described. In Section 5 through simulations the proposed protocol is evaluated and compared it with a LEACH [15] protocol. Finally in Section 6 conclusion is done.

\section{RELATED WORK}

Energy efficient clustering protocols for WSNs have been widely published. The primary goal of clustering is to efficiently manage the energy consumption of among the sensor nodes using multi-hop communication within a particular cluster and by performing data aggregation and fusion in order to decrease the number of transmitted packets to the base station. The cluster formation is basically on the residual energy of sensor nodes and sensor proximity to the CHs.

Linked cluster algorithm (LCA) [13] is among the early ones on clustering of wireless networks. The main focus is on forming an efficient network topology to handle the mobility of nodes. By clustering, $\mathrm{CHs}$ are hoped to form a backbone network to which cluster members can connect while on the move. The primary goal of the proposed distributed algorithm is to form clusters such that a $\mathrm{CH}$ is directly connected to all nodes in its cluster. LCA is designed for maximizing network connectivity. The algorithm assumes synchronized nodes and time-based medium access.

Energy-efficient hierarchical clustering (EEHC) [14] is a distributed randomized clustering algorithm that maximizes the lifetime of a network with a large number of sensor nodes. The EEHC algorithm organizes the sensors in a network into clusters with a hierarchy of $\mathrm{CHs}$. The $\mathrm{CHs}$ collect the information from the sensor nodes within their clusters and send an aggregated report through the hierarchy of $\mathrm{CHs}$ to the base station. The EEHC algorithm assumes that communication environment is contention and error free. The energy consumed in network will depend on the probabilities of each sensor node becoming a $\mathrm{CH}$ at each level in the hierarchy and the maximum number of hops allowed between one cluster node and its $\mathrm{CH}$. The optimal clustering parameters are obtained through hierarchical clustering to minimize the total energy consumption in the network. The energy load is evenly distributed among the sensor nodes by periodic rotation of $\mathrm{CHs}$ or triggered as the energy levels of the $\mathrm{CHs}$ fall below a certain threshold.

Low Energy Adaptive Clustering Hierarchy (LEACH) [15] is one of the first and most popular clustering algorithms for WSNs. It forms clusters based on the received signal strength and uses the $\mathrm{CH}$ nodes as routers to the base station. All the data processing such as data fusion and aggregation are locally performed by the $\mathrm{CH}$. LEACH creates clusters by using a distributed algorithm, where nodes make autonomous decisions without any centralized control. Initially a node decides to be a $\mathrm{CH}$ with a probability $p$ and broadcasts its decision. Each non $\mathrm{CH}$ node selects its cluster by choosing the $\mathrm{CH}$ that can be reached using the less communication energy. The role of being a $\mathrm{CH}$ is rotated periodically among the nodes of the cluster in order to equally balance the energy load. The rotation is performed by getting each node to choose a random number between 0 and 1 . A node becomes a $\mathrm{CH}$ for the current round if the number is less than threshold. The threshold value is calculated as follows:

$$
T(t)= \begin{cases}\frac{p}{1-p^{*} r \bmod \left(\frac{1}{p}\right)} & \text { if } i \in G \\ 0 & \text { otherwise }\end{cases}
$$

where $p$ is the desired percentage of $\mathrm{CH}$ nodes, $r$ is the current round number, and $G$ is the set of nodes that have not been $\mathrm{CHs}$ in the last $1 / p$ rounds. The algorithm uses probabilistic approach to elect $\mathrm{CHs}$; there is a chance that a node with very low energy gets selected as a $\mathrm{CH}$. When this node dies, the whole cell becomes dysfunctional. Also, algorithm uses direct communication to reach base station. This is not always a realistic assumption.

Power-Efficient Gathering in Sensor Information Systems (PEGASIS) [16] is a chain based protocol and it an extension of LEACH protocol. The protocol assumes that all the sensors 
have global knowledge about the network, particularly, the locations of the sensors, and uses a greedy approach. The chain formation starts with the farthest sensor to sink to guarantee that sensors farther away from the sink have close neighbors. The protocol randomly selects only one $\mathrm{CH}$ node from that chain to transmit data to the base station. The data is gathered and moves from node to node, aggregated and eventually sent to the base station by $\mathrm{CH}$. In this protocol, when a sensor fails or dies due to low battery power, the chain is constructed using the same greedy approach by bypassing the failed sensor.

In Distributed Weight-Based Energy-Efficient Hierarchical Clustering (DWEHC) [17], each sensor calculates its weight after locating the neighboring nodes in its area. The weight is a function of the sensor's residual energy and the proximity to the neighbors. In a neighborhood, the node with largest weight would be elected as a $\mathrm{CH}$ and the remaining nodes become members. These nodes are considered as first-level members since they have a direct link to the $\mathrm{CH}$. A node progressively adjusts such membership in order to reach a $\mathrm{CH}$ using the least amount of energy. Basically, a node checks with its non-CH neighbors to find out their minimal cost for reaching a $\mathrm{CH}$. Given the node's knowledge of the distance to its neighbors, it can assess whether it is better to stay a firstlevel member or become a second-level one; reaching the $\mathrm{CH}$ over a two-hop path. The process continues until nodes settles on the most energy efficient intra-cluster topology. To limit the number of levels, every cluster is assigned a range within which member nodes should lay.

In [18], a new clustering algorithm is proposed based on the optimum parameters, which aims at reducing the energy consumption for inter-cluster and intra-cluster communication. The algorithm divides all the nodes into static clusters with optimum parameters. The clusters closer to the base station are smaller in size than those farther from base station. The different sizes of the clusters assure that the cluster heads closer to the BS have enough energy to transmit the fused data which comes from other cluster heads farther away from the base station, which realizes the energy consumption balance in inter-cluster. In intra-cluster, a new mechanism where the current cluster head continuously acts as the local control center is adapted to reduce the frequency of updating cluster and the energy consumption for new cluster head set-up.

In Energy Balanced Fixed Clustering (EBFC) [19] protocol, the sensor area is divided into fixed number of static clusters using the metric transmission range. The node clustering is rotated at regular interval predefined by the protocol. The energy consumption due to intra-cluster communication is reduced by selecting the next cluster heads by cluster heads of current round. This eliminates the negotiation required among sensor nodes to select the cluster head, thereby reducing power consumption and hence extends the network lifetime.

\section{SYSTEM MODEL}

\subsection{Energy Model}

In wireless communication, attenuation of transmitted power decreased exponentially with the increasing transmission distance. Two types of channel transmission models are proposed in [15], i.e., free space model and multi-path attenuation model. When distance $d$ between transmitting node and receiving node is smaller than a certain value $d_{0}$, the free space model is applied with sending power decreasing exponentially by $d^{2}$. Otherwise, the multi-path attenuation model is employed with sending power decreasing exponentially by $d^{4}$.

A simple radio model used in [15] is employed in this paper is shown in Figure 1. The power required to transmits a $k-$ bit message a distance $d$ is given by

$$
E_{T x}(k, d)=\left\{\begin{array}{lr}
E_{\text {elec }} * k+\varepsilon_{\text {fs }} * k * d^{2} & d<d_{0} \\
E_{\text {elec }} * k+\varepsilon_{\text {amp }} * k * d^{4} & d \geq d_{0}
\end{array}\right.
$$

and to receive $k$-bit message, the radio expends

$$
\begin{gathered}
E_{R x}=E_{\text {elec }} * k \\
d_{0}=\sqrt{\frac{\varepsilon_{f s}}{\varepsilon_{\text {amp }}}}
\end{gathered}
$$

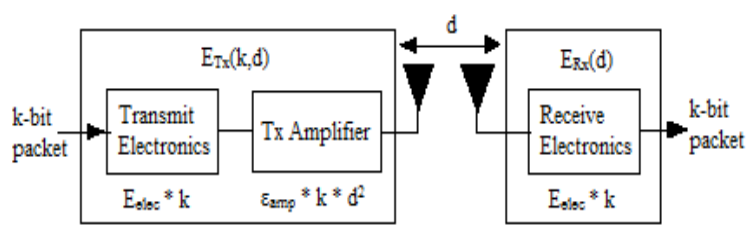

Figure 1. Radio model of sensor node

where $\varepsilon_{f s}$ and $\varepsilon_{a m p}$ are the energy consumption factor of amplification in two radio models, $E_{\text {elec }}$ is the power dissipated per bit to run the transmitter or receiver circuit, $d_{0}$ is the threshold transmission distance for the amplification circuit. The $E_{\text {elec }}$ energy depends on several factors such as digital coding, modulation, filtering and spreading of the signal.

In addition, energy can also be lost during data signal processing such as aggregating, in which $E_{D A}$ denotes energy loss for merging single data signal. The transmitting and receiving a message is not a low cost operation, an inefficient use of the available energy leads to poor performance and short life cycle of the network. Energy in these sensors is a scarce resource and must be managed in an efficient manner. 


\subsection{Network Model}

It is assumed that a WSN composed of large number of ' $\mathrm{N}$ ' sensor nodes randomly deployed in a circular area to collect information periodically. The base station is located at the center of the sensor field and provided with directional antenna. The following assumptions are made on the nature of the WSNs:

a. The base station and nodes in the network are fixed

b. Each node has unique ID

c. Sensor nodes are location aware

d. Each node and base station are capable of controlling transmit power

e. All the nodes in the network are in the communication range of base station

f. Intra-cluster communication is single-hop

\section{SBMC PROTOCOL OPERATION}

Sector Based Multi-hop Clustering is a distributive clustering protocol except the initial round for WSNs. It is used to gather data from randomly deployed sensor nodes and transmit aggregated data to base station. It is designed to support applications where periodic monitoring is required.

A WSNs usually composed of large number of sensor nodes in a given geographical region and the base station is located at the center of the sensor field. Partitioning the entire network into clusters would minimize the energy required for the data dissemination to the base station using multi-hop communication. The protocol operation is divided into three phases: Partition phase, Cluster head selection phase and Routing phase as indicated in Figure 2.

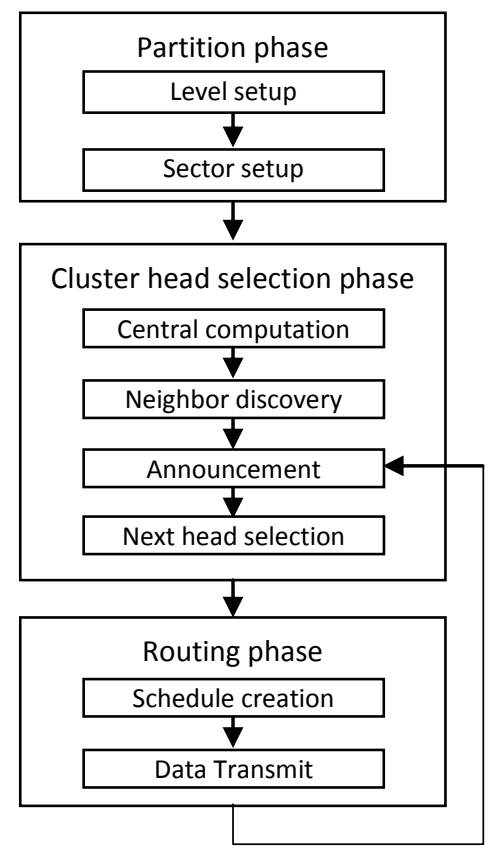

Figure 2. The phases of the proposed SBMC protocol

\subsection{Partition Phase}

During this phase base station wisely organizes the network into unequal clusters by dividing the sensor field into levels and sectors. The base station calculates the optimal number of clusters for the network and creates the required number of levels and sectors. The cluster is an area under curved strip formed by the intersection of circular levels and a triangular section. The clusters nearer to the base station have smaller size than those farthest from the base station. Since the cluster head near to the base station have more data traffic from the lower layers and can able to handle the data for long time. This phase is not repetitive and carried out once at the beginning of the protocol operation. Partition phase consist of two stages: level setup stage and sector setup stage.

\subsubsection{Level setup stage}

During this phase, the base station divides network into concentric circular tracks by transmitting signals at different power levels. The number of levels to be formed is determined initially and depends on the parameters such as number of sensor nodes, their distribution and location of the base station. Initially, the base station broadcast a Level_ID_Packet (LIP) into the network for level 1 with minimum power level. All the nodes that receive this packet set their level 1. Next the base station increases its signal power to reach the next level and broadcast a LIP for level 2 . All the nodes that receive this packet and those nodes that have not set their level ID previously will set their level to 2 . This process continues till the base station sends packets corresponding to all the levels. The level nearest to the base station is top level and the one farthest from it is lower level.

\subsubsection{Sector Setup Stage}

After Leveling is completed, the base station divides the network into equiangular sectors using directional antenna in clockwise or anti clock wise direction. The base station broadcast a Sector_ID_Packet (SIP) into the network for sector 1 in a particular direction. All the nodes that receive this packet set their sector ID 1. Next the base station rotates its antenna direction by sector angle $(\theta)$ and broadcast SIP for sector 2. All the nodes that receive this packet and those nodes that have not set their sector ID previously will set their sector ID to 2. This process continues till the base station sends packets corresponding to all the sectors.

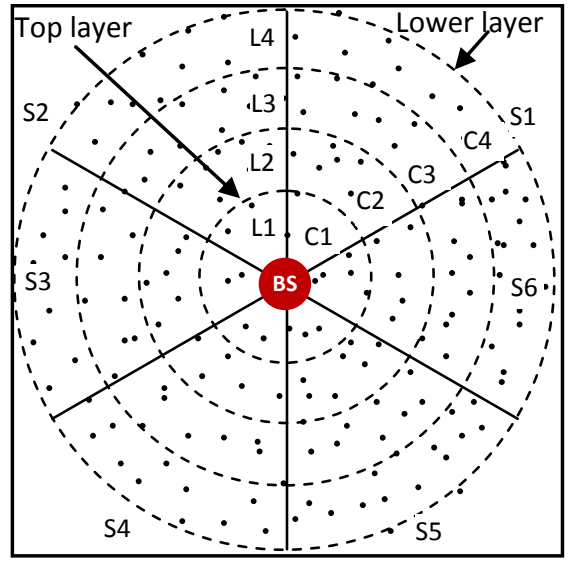

Figure 3. Formation of levels and sectors

At the end of partition phase, sensor network is divided into number of clusters. In the Figure 3, the network consists of 
four levels (i.e. L1through L4) and six sectors (i.e. S1 through S6). Each cluster in the network is identified by level and sector ID (i.e. [LevelID][SectorID]). For example, the cluster $\mathrm{C} 1$ belongs to level 1and sector 1 has the ID [L1][S1] and so on.

\subsection{Cluster Head Selection Phase}

Cluster head is responsible for gathering all the sensed data from the member nodes of the cluster, aggregating data and transmit the aggregated data to the base station. This phase consist of central computation stage, neighbor discovery stage, announcement stage, next head selection stage.

\subsubsection{Central Computation Stage}

During this stage, the base station broadcast a $R E Q$ packet into the network requesting the each nodes information. Upon receiving this, all the nodes in the network send their level ID, sector ID, node ID, energy level and location information to the base station. Based on the level, sector and location information, the base station computes the distance table for each cluster, which has distance between the nodes in the particular cluster and cluster center. In addition, the base station also selects the cluster head for the initial round by taking energy, number of neighbor's and how close to the center of the cluster into account. Next the base station broadcast $C H \_T A B \_C E N T$ message into the network sector by sector. The $C H \_T A B_{-} C E N T$ has initial cluster head IDs, distance table with node coordinates and the $\mathrm{x}$-y coordinate of the center of all clusters in that sector.

\subsubsection{Neighbor Discovery Stage}

During this stage, each node in the cluster extracts their cluster center and initial cluster head ID. If the node is not a cluster head, it will wait for an announcement. Each node maintains a 1-hop neighbor table by extracting the information in the distance table. Later this neighbor table is updated if any of the node energy falls below the minimum threshold value by sending its neighbor's list.

\subsubsection{Announcement Stage}

In this stage, cluster head node broadcast a $C H \_A D V$ message, which has node ID and its location into the network. On hearing this, node clustering is initiated with the farthest node in the cluster. The farthest nodes send JOIN_CH message which has node IDs and residual energy to the nearest neighbors in the direction of cluster head. Next these nodes add their ID and energy level to the received signal and forward to the next neighbor's. This process continues till all the messages reach the cluster head.

\subsubsection{Next Head Selection Stage}

In this stage, based on the residual energy, location and number of neighbor's, current cluster heads selects next heads. This does not require further negotiation to elect cluster heads. These next nodes become the cluster head in the next round.

\subsection{Routing Phase}

During this phase, the cluster heads creates and distributes the TDMA slots for each member of the cluster. Each member nodes of the cluster transmit data during their time slot. The cluster head node aggregates data received from its member nodes and forward it to the base station. This phase consist of schedule creation stage, data transmit stage and re-cluster stage.

\subsubsection{Schedule Creation Stage}

After the completion of node clustering, the cluster heads knows about member nodes of the cluster. Based on the number of nodes, the cluster head assign a unique TDMA time slot to each node in the cluster telling when it can transmit the data. The cluster head broadcast this TDMA time slot along with the next head ID to all the member nodes of the cluster. Member nodes of the cluster can enter into sleep mode till their turn thus minimizing the energy consumption.

\subsubsection{Data Transmit Stage}

After the TDMA schedule is fixed, the data collection begins. The farthest nodes send data to the nearest neighbor's, which adds its data and send to the next neighbor towards the cluster head. This process continues till the data reaches cluster head. After receiving data from all the member nodes, head nodes aggregates and data transmission to the base station is initiated by cluster heads in the lower level using multi-hop communication. When a cluster heads in level $L i$ receives data from the heads in level $L_{i+1}$, it fuses its data with the received one if it is from lower level of the same sector, otherwise discards it. This process continues till the data from the cluster heads of all the levels reaches the base station.

This is the routing phase of SBMC protocol. After a certain time, which is determined a priori, re-clustering of nodes in the next round begins with the next heads by announcing the CH_ADV message as described in the section 4.2.3.

Table 1 Simulation parameters

\begin{tabular}{|l|c|l|}
\hline \multicolumn{1}{|c|}{ Parameter } & Symbol & \multicolumn{1}{c|}{ Values } \\
\hline $\mathrm{T}_{\mathrm{x}} / \mathrm{R}_{\mathrm{x}}$ electronic constant & $E_{\text {elec }}$ & $50 \mathrm{~nJ} / \mathrm{bit}$ \\
\hline \multirow{2}{*}{ Amplifier constant } & $\varepsilon_{f s}$ & $10 \mathrm{pJ} / \mathrm{bit} / \mathrm{m}^{2}$ \\
\cline { 2 - 4 } & $\varepsilon_{\text {amp }}$ & $0.0013 \mathrm{pJ} / \mathrm{bit} / \mathrm{m}^{4}$ \\
\hline Initial Energy & $E_{o}$ & $0.5 \mathrm{joule}$ \\
\hline Energy for Data & $E_{D A}$ & $5 \mathrm{~nJ} / \mathrm{bit} / \mathrm{signal}$ \\
\hline Minimum threshold energy & $E_{\text {THMIN }}$ & $10^{-4} \mathrm{~J}$ \\
\hline
\end{tabular}

\section{RESULT AND DISCUSSION}

The performance comparison of proposed SBMC protocol with LEACH is presented in this section. Simulation experiments are carried out using MATLAB. The simulated WSNs consist of 200 nodes randomly deployed within a circular area of radius 50metres. The base station is located at the center of the field. The most of the parameters and the energy dissipation model of radio and hardware are similar to those in [15]. The simulations are conducted for 4-levels and 6 -sectors, the data packet size was fixed at 500 bytes, and message packet size was set to 25 bytes. The simulation parameters are summarized in Table 1. 
Lifetime is the important criterion for evaluation of performance of sensor networks. In our simulation, we measure the lifetime using three metrics: number of nodes alive over a simulation rounds, first node dies (FND) and last node dies (LND). The proposed SBMC protocol performance is evaluated with LEACH. The first metric number of nodes alive over a simulation rounds gives an idea of the rounds over which the network can send the data before all the nodes in the network die.

In LEACH cluster distribution is not uniform, results in larger variance in energy consumption by all nodes. On the other hand, in SBMC, the cluster heads are distributed uniformly in every sector. As a result, energy consumption is balanced among sensor nodes. This feature helps to prolong the lifetime of the network.

Figure 4 shows the number of sensor nodes that alive after each round for LEACH and SBMC. The result shows that nodes die quicker in LEACH than in proposed SBMC protocol. It is shown from the result that the proposed SBMC protocol outperforms LEACH and prolongs the network life time by $13 \%$.

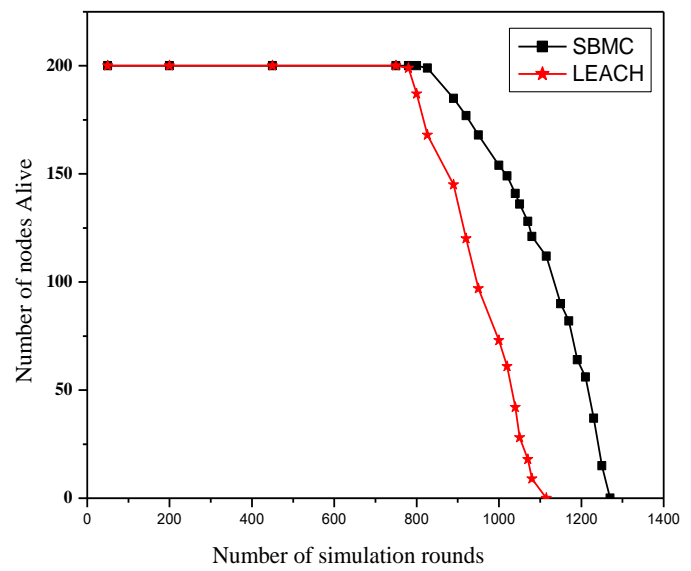

Figure 4. Number of sensor nodes Alive over simulation rounds

Figure 5 and Figure 6 show the network lifetime using the metrics first node dies (FND) and last node dies (LND). It is observed that FND in LEACH is reported earlier than proposed SBMC protocol. Since LEACH uses direct communication to transmit data to the base station, which is more energy expensive. The proposed SBMC uses multi-hop communication for data transmission, which minimizes the energy consumption among the sensor nodes. The Figure 6 shows that proposed protocol has higher network longevity than the LEACH.

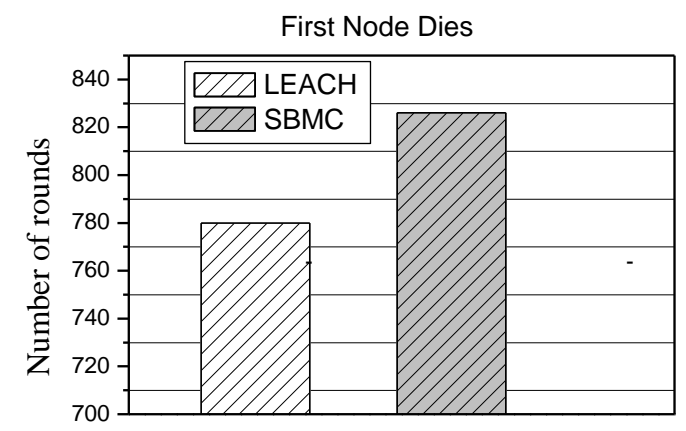

Figure 5. Network lifetime in terms of FND

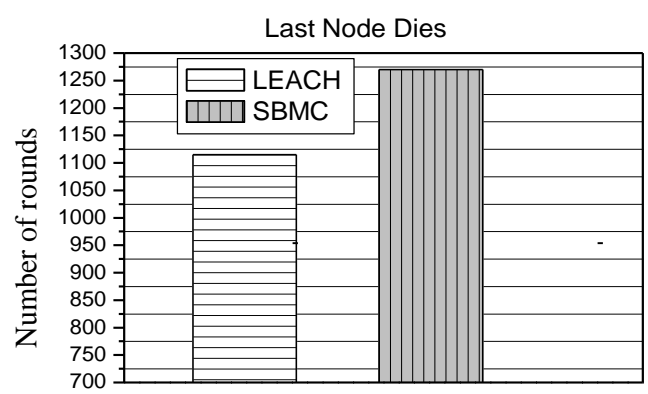

Figure 6. Network lifetime in terms of FND

\section{CONCLUSIONS}

In this paper a novel sector based multi-hop clustering protocol is proposed for energy efficient routing in WSN. The protocol divides the network into levels and sectors and a node clustering is carried out in multi-hop mode. Each node transmits data to cluster head using neighboring nodes, thus minimizing the power consumption. The concept of next head selection reduces the communication overhead required for the selection of cluster heads. The simulation results indicate that SBMC outperforms existing LEACH in terms of network longevity and low energy consumption.

\section{REFERENCES}

[1] R. Szewczyk, E. Osterweil, J. Polastre, M. Hamilton, A. Mainwaring, D. Estrin, "Habitat monitoring with sensor networks," Communications of the ACM, 47 (6) (2004) 34_40.

[2] Y.-C. Tseng, M.-S. Pan, Y.-Y. Tsai, "Wireless sensor networks for emergency navigation," IEEE Computer Society, vol. 39, no. 7, pp. 55-62, 2006.

[3] P.K. Biswas, S. Phoha, "Self-organizing sensor networks for integrated target surveillance," IEEE Transactions on Computers, 55 (8) (2006), pp.1033_1047.

[4] I.F. Akyildiz, et al., "Wireless sensor networks: A Survey,” Computer Networks, 38 (2002), 393-422.

[5] Chong CY, Kumar SP, "Sensor networks: evolution, opportunities, and challenges," In: Proceedings of the IEEE, vol. 91, no. 8; 2003. pp. 1247-56. 
[6] Younis M, Munshi P, Gupta G, Elsharkawy SM, "On efficient clustering of wireless sensor networks," In: Proceedings of the second IEEE workshop on dependability and security in sensor networks and systems; 2006, pp.78-91.

[7] Arboleda LMC, Nasser N. "Comparison of clustering algorithms and protocols for wireless sensor networks," In: Proceedings of the Canadian conference on electrical and computer engineering, 2006. pp. 1787-92.

[8] T. Voigt, H. Ritter, J. Schiller, "Solar-aware routing in wireless sensor networks," In: Ninth IEEE Symposium on Computers and Communications, vol. 1, 2004, pp. 238-243.

[9] S.D. Muruganathan, D.C.F. Ma, R.I. Bhasin, A.O. Fapojuwo, "A centralized energy-efficient routing protocol for wireless sensor networks," IEEE Radio Communications, 43 (3), 2005, pp.8-13.

[10] N. Israr, I. Awan, "Multihop clustering algorithm for load balancing in wireless sensor networks," Internat. $J$. Simul. Systems Sci. Technol, 8 (1), 2007, pp.13-25.

[11] K. Akkaya, M. Younis, "A survey on routing protocols for wireless sensor networks," Elsevier Journal of Ad Hoc Networks, 3 (3), 2005, pp.325-349.

[12] A. Abbasi, M. Younis, "A survey on clustering algorithms for wireless sensor networks," Elsevier J. Computer Communication, 30, 2007, pp. 2826-2841.

[13] D.J. Baker, A. Ephremides, J.A. Flynn, "The design and simulation of a mobile radio network with distributed control," IEEE Journal on Selected Areas in Communications, 1984, pp. 226-237.

[14] S. Bandyopadhyay, E. Coyle, "An energy efficient hierarchical clustering algorithm for wireless sensor networks," In: Proceedings of the 22nd Annual Joint Conference of the IEEE Computer and Communications Societies (INFOCOM 2003), San Francisco, California, April 2003.

[15] W.B. Heinzelman, A.P. Chandrakasan, H. Balakrishnan, "Application specific protocol architecture for wireless microsensor networks," IEEE Transactions on Wireless Networking, 1(4), 2002, pp.660-670.

[16] S. Lindsey, C.S. Raghavendra, "PEGASIS: power efficient gathering in sensor information systems," In: Proceedings of the IEEE Aerospace Conference, Big Sky, Montana, March 2002.

[17] P. Ding, J. Holliday, A. Celik, "Distributed energy efficient hierarchical clustering for wireless sensor networks," In: Proceedings of the IEEE International Conference on Distributed Computing in Sensor Systems (DCOSS'05), Marina Del Rey, CA, June 2005.

[18] Xiang Min, Shi Wei-ren, Jiang Chang-jiang, Zhang Ying, "Energy efficient clustering algorithm for maximizing lifetime of wireless sensor networks," International Journal of Electronics and Communication. (AEÜ) 64 (2010) 289-298.

[19] Boregowda S.B, Babu N.V, Puttamadappa C, and H.S Mruthyunjaya "Energy Balanced Fixed Clustering protocol for Wireless Sensor Networks", International Journal of Computer Science and Network Security, VOL.11 No.8, August 2011, PP. 166-172.

\section{AUTHORS PROFILE}

Bore Gowda S. B received B.E. degree in Electronics and Communication from Mysore University and MTech from Visvesvaraya Technological University, Karnataka. He is currently serving as Assistant Professor (Selection Grade) in the Department of Electronics and Communication Engineering, Manipal Institute of Technology, Manipal, He is pursuing Doctoral degree at Manipal University, India. His main research interests include Ad-Hoc and wireless sensor networks and embedded system desi

Dr C Puttamadappa obtained his B.E degree from Mysore University, PG degree from Bangalore University and Doctral Degree from Jadavpur University, Kolkatta. He is working as Principal, Sapthagiri college of Engineering, Bangalore. His research area of interest is Devices \& Networks

H. S. Mruthyunjaya has completed his bachelor degree in Electronics and Communication Engineering from Mysore University in 1988 and obtained his master's degree in Electronics and Control Systems Engineering from Birla Institute of Technology and Science, Pilani in 1994. He has a $\mathrm{Ph} . \mathrm{D}$ in Electronics and Communication Engineering conferred by Manipal University. He is currently serving as a Professor in the Department of Electronics and Communication Engineering, Manipal Institute of Technology, Manipal, His areas of major interests are the Optical Fiber Communication systems, Fiber Optics, Photonic Crystal Fibers, WDM networks and systems, Electromagnetic theory \& General areas of Digital Communication Systems.

Mr Babu $\boldsymbol{N} \boldsymbol{V}$ obtained his B.E degree from Visveswaraya technological University, Belagum and PG from Dr MGR University, Chennai. He is working as Assistant Professor in E\&CE department, SJBIT, Bangalore. Currently he is pursuing Ph.D under the guidance of Dr Puttamadappa $\mathrm{C}$ from Kuvempu University, Shimoga. 